\title{
A generalization of Wolstenholme's Theorem
}

\author{
Yali $\mathrm{Su}^{1}$ Jiming $\mathrm{Yang}^{2}$ and Shuying $\mathrm{Li}^{3}$ \\ ${ }^{1}$ School of Information Technology and Engineering, Yuxi Normal University, Yuxi, Yunnan, China- 653100. \\ ${ }^{2}$ School of Science, Yuxi Normal University, Yuxi, Yunnan, China- 653100. \\ ${ }^{3}$ College of Resource and Environment, Yuxi Normal University, Yuxi, Yunnan, China- 653100.
}

yxshuyali@yahoo.com.cn

\begin{abstract}
A generalization of Wolstenholme's Theorem is given.

Keywords: Wolstenholme's Theorem; prime number; residue
\end{abstract}

\section{Introduction}

In number theory, there is a well-known theorem about prime numbers, it is Wolstenholme Theorem. Let prime number $p>3$, Let $\frac{1}{S}$ satisfy:

$$
s s^{*} \equiv 1\left(\bmod p^{2}\right)
$$

$s^{*}$ is an integer,then

$$
1+\frac{1}{2}+\frac{1}{3}+\cdots+\frac{1}{p-1} \equiv 0\left(\bmod p^{2}\right) \text {. }
$$

This theorem lead to a number of Chinese and foreign scholars to study it with a strong interest. It has been generalized to many forms.

Zhang shusheng(1989) pointed out generalization of Wolstenholme's Theorem of the following Proposition 1.

Proposition 1 Let prime number $p>3$, Let $\frac{1}{s}$ satisfy:

$s s^{*} \equiv 1\left(\bmod p^{2}\right)$

$s^{*}$ is an integer,then

$$
\sum_{k=1}^{p-1} \frac{1}{t p+k} \equiv 0\left(\bmod p^{2}\right)
$$

In the expressions, $t$ is any integer.

Chen Keyin(2005) pointed out the following Proposition 2, which is equivalent with the theorem of Wolstenholme.

Proposition 2 Let prime number $p>3$, then

$$
\sum_{k=1}^{p-1} k^{p(p-1)-1} \equiv 0\left(\bmod p^{2}\right) \text {. }
$$

At the same time, Chen Keyin also made the promotion of Proposition 2 and obtain the following Proposition 3.

Proposition 3 Let prime number $p>3$, $\mathrm{n}$ is an odd number greater than $1, p-1$ is not divisible by $n-1, \mathrm{t}$ is any integer,then

$$
\sum_{k=1}^{p-1}(t p+k)^{n} \equiv 0\left(\bmod p^{2}\right)
$$

Zeng denggao(2004) pointed out generalization of Wolstenholme's Theorem, it is the following Proposition 4.

Proposition $4 \mathrm{p}$ is a prime number

$p>3, \nu, t_{0}, t_{k} \in Z(1 \leq k \leq p-1)$

$0 \leq 2 \nu<p-3,\left(t_{0}, p\right)=1$

$t_{1}+t_{p-1}=t_{2}+t_{p-2}=\cdots=t_{\frac{p-1}{2}}+t_{\frac{p-1}{2}}$.

$s^{*}$ is an integer and Let $s^{*}$ satisfy:

$s s^{*} \equiv 1\left(\bmod p^{2}\right)$,then

$\sum_{k=1}^{p-1} \overline{\left(t_{k} p+k t_{0}\right)^{2 \nu+1}} \equiv 0\left(\bmod p^{2}\right)$.

Hong Shaofang(2007) mentioned generalization of Wolstenholme's Theorem, it is the following Proposition 5.

Proposition 5 Let $\mathrm{m}$ and $\mathrm{n}$ is any integer , $m \geq 0, n \geq 1,\langle n\rangle=\{1, \cdots, n\} \cdot p_{1}, \cdots, p_{n}$ are different prime numbers and all greater than 3 ,then 


$$
\sum_{\substack{j=1 \\ \forall i \in\langle n\rangle,\left(j, p_{i}\right)=1}}^{p_{1} \cdots p_{n}} \frac{1}{m p_{1} \cdots p_{n}+j}
$$

In the expressions, molecules of fraction can be divisible by $p_{1}^{2} \cdots p_{n}^{2}$.

\section{New generalization of Wolstenholme's Theorem}

This paper describes another generalized form of Wolstenholme's Theorem, It is the following theorem.

Theorem Let prime number $p>3$, $\mathrm{t}$ is any integer, $\mathrm{r}$ is a non-negative integer, $p^{r} \mid 2 t+1$. Let $\frac{1}{s}$ satisfy:

$$
s s^{*} \equiv 1\left(\bmod p^{r+2}\right)
$$

$s^{*}$ is an integer,then

$$
\sum_{k=1}^{p-1} \frac{1}{t p+k} \equiv 0\left(\bmod p^{r+2}\right) \text {. }
$$

when $t=0, r=0$, it is Wolstenholme theorem.

\section{Proof}

Lemma Let prime number $p>3$, then

$$
\sum_{i=1}^{\frac{p-1}{2}} \prod_{\substack{j=1 \\ j \neq i \\ j \neq p-i}}^{p-1} j \equiv 0(\text { m o d } p) \text {. }
$$

Proof (1) multiplied by $(p-1)$ ! on both sides, can be get:

$\sum_{i=1}^{p-1} \prod_{\substack{j=1 \\ j \neq i}}^{p-1} j \equiv 0\left(\right.$ m o d $\left.p^{2}\right)$.

But

$$
\begin{aligned}
& \sum_{i=1}^{p-1} \prod_{\substack{j=1 \\
j \neq i}}^{p-1} j=\sum_{i=1}^{\frac{p-1}{2}}\left[\prod_{\substack{j=1 \\
j \neq i}}^{p-1} j+\prod_{\substack{j=1 \\
j \neq p-i}}^{p-1} j\right] \\
& =\sum_{i=1}^{\frac{p-1}{2}}\left[(p-i) \prod_{\substack{j=1 \\
j \neq i \\
j \neq p-i}}^{p-1} j+i \prod_{\substack{j=1 \\
j \neq i \\
j \neq p-i}}^{p-1} j\right]=p \sum_{i=1}^{\frac{p-1}{2}} \prod_{\substack{j=1 \\
j \neq i \\
j \neq p-i}}^{p-1} j .
\end{aligned}
$$

By (9), (10), we can get (8) holds.

Easy to obtain (11) from the proof of the theorem.

$$
\begin{aligned}
& \sum_{i=1}^{p-1} \prod_{\substack{j=1 \\
j \neq i}}^{p-1}(t p+j)=\sum_{i=1}^{\frac{p-1}{2}}\left[\prod_{\substack{j=1 \\
j \neq i}}^{p-1}(t p+j)+\prod_{\substack{j=1 \\
j \neq p-i}}^{p-1}(t p+j)\right] \\
& =\sum_{i=1}^{\frac{p-1}{2}}\left[(t p+p-i) \prod_{\substack{j=1 \\
j \neq i \\
j \neq p-i}}^{p-1}(t p+j)+(t p+i) \prod_{\substack{j=1 \\
j \neq i \\
j \neq p-i}}^{p-1}(t p+j)\right] \\
& =p(2 t+1) \sum_{i=1}^{\frac{p-1}{2}} \prod_{\substack{j=1 \\
j \neq i \\
j \neq p-i}}^{p-1}(t p+j) .
\end{aligned}
$$

Obtained (12) by the Lemma.

$$
\begin{aligned}
& \sum_{i=1}^{\frac{p-1}{2}} \prod_{\substack{j=1 \\
j \neq i \\
j \neq p-i \\
p-1}}^{p-1}(t p+j) \\
& \equiv \sum_{i=1}^{\frac{p-1}{2}} \prod_{\substack{j=1 \\
j \neq i \\
j \neq p-i}}^{p-1} j \equiv 0(\bmod p) .
\end{aligned}
$$

From the known conditions $p^{r} \mid 2 t+1$,(11)and (12), we can get:

$$
\sum_{i=1}^{p-1} \prod_{\substack{j=1 \\ j \neq i}}^{p-1}(t p+j) \equiv 0\left(\bmod p^{r+2}\right) \text {. }
$$

By (13), can be get:

$$
\begin{aligned}
& \left(\prod_{j=1}^{p-1}(t p+j)\right) \sum_{i=1}^{p-1}(t p+i)^{*}=\sum_{i=1}^{p-1}(t p+i)^{*} \prod_{j=1}^{p-1}(t p+j) \\
& \equiv \sum_{i=1}^{p-1} \prod_{\substack{j=1 \\
j \neq i}}^{p-1}(t p+j) \equiv 0\left(\bmod p^{r+2}\right) \cdot \\
& \operatorname{But}\left(\prod_{j=1}^{p-1}(t p+j), p^{r+2}\right)=1, \text { by (14), we can }
\end{aligned}
$$

get:

$\sum_{i=1}^{p-1}(t p+i)^{*} \equiv 0\left(\bmod p^{r+2}\right)$.

By (15), we can get (7) holds. 


\section{References}

1. Bayat MA (1997) Generalization of Wolstenholme's Theorem, Amer. Math. Monthly. pp: 557-560.

2. Chen Keyin (2005) Another equivalent form of Theorem Wolstenholme. Math. in Practice \& Theory. 3, 71-74.

3. Hardy GH and Wright EM (1960) An introduction to the Number theory.4th ed, Oxford Univ.Press, London. pp:156-157.

4. Hong Shaofang (2007) A Generalization of Wolstenholme's Theorem. J. South China Normal Univ. 2, 24-28.

5. Hua Luogen (1979) Introduction to Number Theory. Sci. Press, Beijing, China. pp:187-189.

6. Ke shao, Sun qi (2001) Number theory lecture notes. Higher Edu. Press, Beijing, China. 2, 230-241.

7. Sun qi \& Hong shaofang (1999) p-adic proof and Generalization of Wolstenholme's Theorem. J. Sichuan Uni. 5, 840-844.

8. Zeng denggao (2004) A Generalization of Wolstenholme's Theorem, Math. in Practice \& Theory. 3, 89-91.

9. Zhang shushing (1989) A Generalization of number theory theorem, Math. in Practice \& Theory. 1, 86-89. 